\title{
AVALIAÇÃO DA DISPONIBILIDADE DE FERRO EM OVO, CENOURA E COUVE E EM SUAS MISTURAS ${ }^{1}$
}

\author{
Flávia Maria Vasques Farinazzi MACHADO², Solange Guidolin CANNIATTI-BRAZACA ${ }^{3, *}$, \\ Sonia Maria de Stefano PIEDADE ${ }^{4}$
}

\begin{abstract}
RESUMO
A desordem nutricional mais comum no mundo é a deficiência de ferro. Sua disponibilidade é influenciada por outras substâncias. Os objetivos da pesquisa foram: avaliar a disponibilidade de ferro em alimentos fonte deste e de vitamina A, ovo (o), cenoura (c) e couve (cv), e verificar interação do ferro com $\alpha$ e $\beta$-caroteno, e com outros interferentes. As misturas foram de ovo com cenoura e ovo com couve nas proporções $(1: 1),(1: 1,5),(1: 2),(1: 2,5)$ e $(1: 3)$ e 3 alimentos sozinhos, totalizando 13 amostras. As amostras, após preparo, foram submetidas às análises de composição centesimal, ferro total, taninos, ácido fítico, ácido oxálico, $\alpha$-caroteno e $\beta$-caroteno e diálise de ferro. Os dados foram analisados por teste de Tukey e correlação. Foi observada correlação positiva do ferro dialisável em relação à umidade e $\beta$-caroteno e negativa para extrato etéreo, proteínas e cinza. A amostra $1,5 \mathrm{c}$ apresentou teores mais elevados de taninos. As amostras $1 \mathrm{cv}$ e $1,5 \mathrm{cv}$ maiores teores de oxalato e ferro dialisável mais baixo. Pode ser concluído que misturas com maiores proporções de cenoura e couve aumentaram a disponibilidade do ferro e não houve interferência dos fatores antinutricionais. O $\beta$-caroteno apresentou correlação positiva com ferro dialisável, exercendo influência na diminuição do efeito dos fatores antinutricionais.
\end{abstract}

Palavras-chave: cenoura, couve, ovo, disponibilidade, ferro, interação.

\section{SUMMARY}

IRON AVAILABILITY IN EGG, CARROT AND CABBAGE AND IN THEIR MIXTURES. The most common nutritional disorder in the world is a deficiency in iron. The availability of iron is influenced by other substances. The aim of this research is to evaluate the availability of iron in food iron sources and vitamin A, egg (e), carrot (c) and cabbage (cb), as well as to analyse the interaction of the iron with $\alpha$, $\beta$-carotene and with other interferences. The mixtures were egg with carrot and egg with cabbage in the proportions of ( $1: 1)$, (1:1.5), $(1: 2),(1: 2.5)$ and ( $1: 3)$ plus the three food types alone, which totalled 13 food mixtures. After being prepared, the food mixtures were analysed for the centesimal composition, iron, tannin, phytic acid, oxalic acid, $\alpha, \beta$-carotene and in vitro iron dialysability. The data was analysed using Tukey and correlation. A significant positive correlation of in vitro iron dialysability in relation to moisture and $\beta$-carotene, negative for fat, proteins and ash was observed. The mixture of $1.5 \mathrm{c}$ showed high levels of tannins and the mixtures of $1 \mathrm{cb}$ and $1.5 \mathrm{cb}$ high levels of oxalates and lower levels of in vitro iron dialysability in relation to the other mixtures. It can be concluded that the mixtures with larger carrot and cabbage proportions increased the iron availability and there was no interference from the antinutritional factors. The $\beta$-carotene showed a significant positive correlation with in vitro iron dialysability influencing the decrease of the antinutritional factors effect in iron availability.

Keywords: carrot, cabbage, egg, availability, iron, interaction.

\section{1 - INTRODUÇÃO}

A prevalência da desnutrição na população brasileira, em especial no grupo infantil, está diminuindo de forma significativa. O fator agravante, porém, é que o comportamento da anemia ferropriva não acompanha a melhoria do estado nutricional [2].

A deficiência de ferro é o distúrbio nutricional mais comum, atingindo cerca de 4,5 bilhões de pessoas, aproximadamente $74 \%$ da população mundial e 2 bilhões de

\footnotetext{
$\overline{{ }^{1} \text { Recebido para publicação em 19/7/2005. Aceito para publicação em }}$ 6/7/2006 (001579)

"Escola Superior de Agricultura "Luiz de Queiroz",

Universidade de São Paulo

${ }^{3}$ Departamento de Agroindústria, Alimentos e Nutrição,

Escola Superior de Agricultura "Luiz de Queiroz",

Av. Pádua Dias, 11, C. P. 9, CEP 13418-900, Piracicaba (SP), Brasil,

E-mail:sgcbraza@esalq.usp.br

${ }^{4}$ Departamento de Ciências Exatas,

Escola Superior de Agricultura "Luiz de Queiroz",

Universidade de São Paulo

* A quem a correspondência deve ser enviada
}

pessoas, cerca de $30 \%$ da população mundial, são anêmicas. Isso ocorre principalmente devido à deficiência de ferro e, em países em desenvolvimento, freqüentemente exacerbada pela malária e fortes infecções [3, 32].

Estimativas mundiais mostram que 190 milhões de pessoas apresentam deficiência subclínica, 13 milhões, xeroftalmia e, como conseqüência, de 250.000 a 500.000 crianças são acometidas de cegueira irreversível, anualmente [9]. No Brasil, a deficiência de vitamina A é um problema endêmico em grandes áreas das regiões Norte, Nordeste e Sudeste. De acordo com CABALLERO [7], a deficiência de ferro se associa epidemiologicamente com a deficiência de vitamina A.

A biodisponibilidade do ferro, proporção do total ingerido que é absorvida e utilizada, é também influenciada por fatores relacionados ao organismo [21]. LAYRISSE et al. [22] sugerem que a vitamina A previne o efeito inibitório dos polifenóis presentes no chá e café, os quais são responsáveis pela redução de mais de $50 \%$ na absorção do ferro dos alimentos. 
Os carotenóides com atividade pró-vitamínica ( $\beta$-caroteno, $\alpha$-caroteno e $\beta$-criptoxantina) provenientes dos vegetais têm sido considerados como o grupo de substâncias que podem ser transformadas em vitamina A na alimentação humana, contribuindo com $68 \%$ deste nutriente da dieta em termos mundiais [35].

A associação entre o baixo consumo de ferro biodisponível e anemia reflete a composição de refeição imprópria para a manutenção de um adequado estado nutricional de ferro, sendo o consumo de ferro total menos importante do que a combinação dos alimentos na determinação da anemia.

De acordo com o estudo de GERMANO [17], as misturas com ovo aumentaram a disponibilidade do ferro, principalmente pelo conteúdo expressivo de proteína e extrato etéreo. A proteína pode atuar como fator coadjuvante, aumentando a absorção do ferro, e a fibra, como fator limitante em sua absorção. A utilização de misturas com ovo, principalmente ovo e cenoura, na dieta de grupos populacionais carentes em ferro, bem como para outros grupos especiais, visando o aumento da absorção do ferro, é recomendada.

Segundo o IBGE [15], a quantidade de ovos produzidos no Brasil vem aumentando, uma vez que foram produzidas 151.939 toneladas em março de 2002, 152.350 toneladas em março de 2003 e 160.679 toneladas em março de 2004. Para a cenoura e a couve, a produção alcançou valores, em 1996 , de 453.907 e 96.916 toneladas, respectivamente. Segundo dados do CEAGESP [8], a produção e comercialização de cenoura e couve alcançam ofertas estáveis praticamente durante o ano todo, ocorrendo escassez apenas nos meses de fevereiro, junho e julho.

O ovo, a cenoura e a couve são alimentos disponíveis, podendo mesmo serem cultivados com facilidade pela população. Não possuem custos elevados, possibilitando sua aquisição por grande parte da população.

Devido à disponibilidade [17] e custo destes alimentos, da importância do ferro e da vitamina A na nutrição humana e da alta prevalência de suas deficiências na população brasileira e mundial, torna-se necessária a avaliação da disponibilidade do ferro em combinações de alimentos, como também quantificar e verificar as interações que ocorrem entre o ferro e os demais nutrientes, incluindo $\alpha \mathrm{e}$ $\beta$-caroteno. Os alimentos a serem utilizados em diferentes proporções serão o ovo, a cenoura e a couve, sendo o ovo utilizado como fonte de ferro.

\section{2 - MATERIAL E MÉTODOS}

\section{1 - Matéria-prima}

A matéria-prima foi obtida do comércio local. Os alimentos fonte de ferro (ovo) e de $\alpha$ - e $\beta$-caroteno (cenoura e couve) são encontrados no mercado, disponíveis durante o ano todo e apresentam preços baixos.

As cenouras e as folhas de couve, cultivadas sob irrigação, foram colhidas em estágio de maturação ótimo para consumo. Os ovos foram adquiridos frescos provenientes de uma granja de coleta diária.

\section{2 - Métodos}

\subsection{1 - Preparo das amostras}

Foi realizado segundo GERMANO [17], sendo todos os alimentos submetidos à cocção até o ponto de consumo, em utensílios de inox, utilizando-se água destilada em todas as etapas do preparo.

a) Ovo

Os ovos foram colocados em panela de inox com água fria e aquecidos até a temperatura de ebulição. Após 10 min, foram retiradas e removidas as cascas, sendo utilizados para as análises 4,6 kg de ovos.

\section{b) Cenoura (Daucus carota L.)}

As cenouras foram lavadas, descascadas, cortadas em tiras na espessura de $1 \mathrm{~cm}$, colocadas em água fria e submetidas à cocção em temperatura de ebulição, com panela aberta, na proporção de 1:5 (cenoura:água), por 20 min. A quantidade de cenouras submetidas ao preparo foi de $7,0 \mathrm{~kg}$.

c) Couve manteiga (Brassica oleracea var. acephala L.)

As folhas de couve foram lavadas, desprezando-se os talos, cortadas em tamanho padrão mínimo (aproximadamente $0,5 \mathrm{~cm}$ ) e submetidas à cocção em panela de inox, sem tampa, com água na temperatura de ebulição, na proporção 1:5 (couve:água), por 15 min [17]. A quantidade de folhas de couve utilizadas foi de $2,3 \mathrm{~kg}$.

\subsection{2 - Amostras}

A partir da fonte de ferro (ovo) e de $\alpha$ - e $\beta$-caroteno (cenoura e couve), foram preparadas misturas nas proporções ovo:cenoura e ovo: couve de $1: 1,1: 1,5,1: 2,1: 2,5 \mathrm{e}$ $1: 3$, sendo analisadas tanto as misturas como os alimentos isoladamente, totalizando 13 amostras.

Para as análises de diálise de ferro in vitro, $\alpha$ - e $\beta$-caroteno, as amostras foram homogeneizadas e armazenadas em congelador a $-18{ }^{\circ} \mathrm{C}$, para futuras determinações.

Para as demais análises, as misturas foram homogeneizadas, desidratadas em estufa a $55^{\circ} \mathrm{C}$, moídas em moinhos de facas Marca Marconi modelo especial 60 ciclos, peneiradas a 60 "mesh", acondicionadas em embalagem plástica de polietileno e mantidas refrigeradas à aproximadamente $11^{\circ} \mathrm{C}$. Todas as determinações foram realizadas em triplicata.

\subsection{3 - Composição centesimal}

As análises químicas da matéria seca, cinza, extrato etéreo e proteína foram feitas de acordo com a metodologia descrita pela Associação Oficial de Química Analítica, AOAC [1]. 


\subsection{4 - Ferro}

O teor de ferro total foi determinado pelo método de SARRUGE \& HAAG [38], utilizando digestão nitro-perclórica e leitura em espectrofotômetro de absorção atômica em comprimento de onda de 248,3 nanômetros.

\subsection{5 - Fatores antinutricionais}

- Taninos: Os taninos foram analisados segundo a metodologia descrita por PRICE et al. [33], com leitura a $500 \mathrm{~nm}$ em espectrofotômetro Beckman modelo DU 640 e utilizando como padrão a catequina;

- Ácido fítico: O teor de ácido fítico nas amostras foi determinado segundo o método descrito por GRYNSPAN \& CHERYAN [19], com leitura da absorbância a 500 nm em espectrofotômetro; e

- Ácido oxálico: O ácido oxálico foi determinado pelo método de Moir [27], por titulação, utilizando $\mathrm{KMnO}_{4}$.

\subsection{6 - Pró-vitamina A ( $\alpha$ - e $\beta$ - caroteno)}

As análises de $\alpha$-caroteno e $\beta$-caroteno foram feitas na Universidade Federal de Viçosa, no Departamento de Nutrição, utilizando-se Cromatografia Líquida de Alta Eficiência [18], por meio de um Cromatógrafo Líquido modelo LC 10 AD, Shimadzu, além dos seguintes equipamentos: injetor automático, com loop de $50 \mu \mathrm{L}$; Coluna cromatográfica LiChrospher 100 RP-18 (5 micra), Merck, com 250 mm de comprimento e $4 \mathrm{~mm}$ de diâmetro interno. As leituras foram a $450 \mathrm{~nm}$, em detector espectrofotométrico de arranjos de diodos, modelo SPD-10 AV; Fase móvel: metanol (80): acetonitrila (10): acetato de etila (10); Vazão (fluxo da fase móvel): 2,0 mL/min. As amostras foram analisadas em triplicata, injetando-se de 10 a $50 \mu \mathrm{L}$ de cada amostra na coluna cromatográfica.

O valor de vitamina A foi expresso em Retinol Equivalente (RE)/100 g de amostra e em Atividade de Retinol Equivalente (RAE)/100 g. Conforme NATIONAL ACADEMY OF SCIENCES-NATIONAL COUNCIL RESEARCH [30], $6 \mu \mathrm{g}$ de $\beta$-caroteno e $12 \mu \mathrm{g}$ de $\alpha$-caroteno correspondem a $1 \mu \mathrm{g}$ RE. No entanto, na última recomendação do NATIONAL COUNCIL RESEARCH [31], o Equivalente de Retinol (RE) foi substituído pelo Equivalente de Atividade de Retinol (RAE) e houve mudança nos fatores de conversão de carotenóides, sendo utilizados, atualmente, $24 \mu \mathrm{g}$ de $\alpha$-caroteno, $12 \mu \mathrm{g}$ de $\beta$-caroteno e $1 \mu \mathrm{g}$ de retinol, correspondentes a 1 RAE.

\subsubsection{Diálise de ferro in vitro}

A análise da diálise de ferro foi feita segundo o método proposto por WHITTAKER et al. [45]. As amostras foram homogeneizadas em água deionizada e adicionadas de $\mathrm{HCl} 6 \mathrm{~N}$ até que o pH atingisse valor 2, em seguida, adicionou-se $\mathrm{HCl}$ 0,01 $\mathrm{N}$ até que se completasse o volume de $100 \mathrm{~mL}$. A digestão foi feita pela adição HCl-pepsina com incubação a $37{ }^{\circ} \mathrm{C}$ e agitação a $200 \mathrm{rpm}$ por $2 \mathrm{~h}$. A acidez titulável foi feita pela adição da solução de pancreatina-bile seguida de titulação com KOH 0,5 N até pH 7,5. A partir do volume de KOH titulável, foi feita a diluição de mesmo volume de $\mathrm{NaHCO}_{3}$ 0,5 N.

A diálise foi feita colocando-se o material digerido em sacos de diálise. Foi acrescentado três vezes o volume de $\mathrm{NaHCO}_{3}$ 0,5 $\mathrm{N}$, de modo que o digerido ficasse submerso. Os frascos foram cobertos e agitados durante $30 \mathrm{~min}$ a $37{ }^{\circ} \mathrm{C}$. Foi adicionada suspensão de bile pancreatina, com incubação por mais $2 \mathrm{~h}$. O conteúdo dialisável foi completado a $25 \mathrm{~mL}$, com água deionizada. Em seguida, foram pipetados $5 \mathrm{~mL}$ do dialisado para o tubo de centrífuga com adição de solução precipitante de proteínas. Foi adicionada solução cromogênica ao sobrenadante. Após $10 \mathrm{~min}$, foi feita a leitura a $533 \mathrm{~nm}$ em espectrofotometro. A quantidade de ferro dialisado foi obtida por meio de curva padrão previamente preparada. Os resultados foram expressos em porcentagem.

\subsection{Delineamento experimental}

O delineamento estatístico foi inteiramente casualisado, com 13 tratamentos e três repetições por tratamento (amostra). AMOSTRA 1: ovo e cenoura (1:1); AMOSTRA 2: ovo e cenoura $(1: 1,5)$; AMOSTRA 3: ovo e cenoura $(1: 2)$; AMOSTRA 4: ovo e cenoura $(1: 2,5)$; AMOSTRA 5: ovo e cenoura (1:3); AMOSTRA 6: ovo e couve (1:1); AMOSTRA 7: ovo e couve ( $1: 1,5)$; AMOSTRA 8 : ovo e couve ( $1: 2)$; AMOSTRA 9: ovo e couve $(1: 2,5)$; AMOSTRA 10: ovo e couve ( $1: 3)$; AMOSTRA 11: ovo; AMOSTRA 12: cenoura; e AMOSTRA 13: couve. Os dados foram submetidos ao teste $\mathrm{F}$ da análise de variância e, posteriormente, foi realizado o teste de Tukey para comparar as médias das amostras. Foram feitas as correlações entre a variável disponibilidade de ferro e outras variáveis (umidade, extrato etéreo, proteínas, cinzas, fitatos, oxalatos, taninos e $\beta$-caroteno), em nível de $5 \%$ de probabilidade, para avaliar os efeitos interferentes das diferentes proporções. As análises foram feitas pelo programa estatístico SAS [39].

\section{3 - RESULTADOS E DISCUSSÃO}

\section{1 - Composição centesimal}

Na Tabela 1, encontram-se os valores de umidade, proteína, extrato etéreo, cinzas e carboidratos para as amostras analisadas.

Os valores de umidade encontrados nas amostras de ovo A11(o) foram superiores aos registrados na literatura, pois ENGLERT [12], SALINAS [36] e TORRES et al. [44] encontraram 72,48 g, 73,9 g e 76,7 g/100 g, respectivamente. Os valores para cenoura e couve se apresentaram dentro da normalidade, sendo encontrados na literatura os valores de 95,55 g, 94,48 g e 95,4 g/100 g [12, 13, 41].

Segundo SILVA [41], o teor de umidade nos alimentos pode ser influenciado por fatores que incluem o cultivar, as condições de armazenamento, a época do ano, a idade da planta e o tempo de cocção. Em relação aos ovos, conforme o tempo de armazenamento, a umidade tende a diminuir. Considerando-se as misturas, as amostras com cenoura 
TABELA 1 - Teores de umidade, proteína, extrato etéreo, cinzas e carboidratos, em g/100 g de amostras (base úmida).

\begin{tabular}{|c|c|c|c|c|c|}
\hline \multirow[t]{2}{*}{ Amostras* } & Umidade & $\begin{array}{c}\text { Extrato } \\
\text { etéreo }\end{array}$ & Proteínas & Cinzas & Carboidratos*^ \\
\hline & \multicolumn{5}{|c|}{$(\mathrm{g} / 100 \mathrm{~g})$} \\
\hline $\mathrm{A} 1$ (1 c) & $89,96^{2 \times 3}$ & $2,31^{2 b}$ & $2,68 \pm 0,4^{1 \mathrm{~d}}$ & $0,87^{2 \mathrm{bc}}$ & 4,18 \\
\hline $\mathrm{A} 2(1,5 \mathrm{c})$ & $91,14^{i}$ & $1,61^{\mathrm{e}}$ & $2,44 \pm 0,6^{\mathrm{ef}}$ & $0,81^{\text {cd }}$ & 4,00 \\
\hline A3 (2 c) & $91,96^{f}$ & 1,349 & $2,04 \pm 0,5^{9}$ & $0,80^{\text {de }}$ & 3,86 \\
\hline A4 (2,5 c) & $92,49^{d}$ & $1,14^{\mathrm{h}}$ & $1,53 \pm 0,5^{h}$ & $0,79^{\text {de }}$ & 4,05 \\
\hline A5 (3 c) & $92,91^{\mathrm{c}}$ & $0,96^{i}$ & $1,48 \pm 0,4^{\mathrm{h}}$ & $0,75^{\mathrm{e}}$ & 3,90 \\
\hline A6 (1 cv) & $89,53^{\prime}$ & $2,18^{\mathrm{c}}$ & $3,17 \pm 0,8^{b}$ & $0,97^{a}$ & 4,15 \\
\hline A7 $(1,5 \mathrm{cv})$ & $90,54^{j}$ & $1,86^{d}$ & $2,95 \pm 0,4^{c}$ & $0,97^{a}$ & 3,68 \\
\hline A8 $(2 \mathrm{cv})$ & $91,38^{\mathrm{h}}$ & $1,48^{\mathrm{f}}$ & $2,57 \pm 0,4^{\text {de }}$ & $0,96^{\mathrm{a}}$ & 3,61 \\
\hline A9 $(2,5 \mathrm{cv})$ & $91,8^{9}$ & $1,28^{g}$ & $2,39 \pm 0,3^{f}$ & $0,96^{a}$ & 3,51 \\
\hline A10 (3 cv) & $92,26^{e}$ & $1,13^{h}$ & $2,18 \pm 0,6^{g}$ & $0,91^{\mathrm{ab}}$ & 3,52 \\
\hline $\mathrm{A} 11$ (o) & $83,93^{m}$ & $5,94^{a}$ & $7,22 \pm 0,1^{a}$ & $0,65^{a}$ & 2,26 \\
\hline $\mathrm{A} 12$ (c) & $95,74^{\mathrm{a}}$ & $0,21^{\mathrm{k}}$ & $0,55 \pm 0,2^{j}$ & $0,57^{j}$ & 2,93 \\
\hline $\mathrm{A} 13(\mathrm{cv})$ & $94,94^{b}$ & $0,33^{j}$ & $1,10 \pm 0,4^{i}$ & $0,74^{i}$ & 2,89 \\
\hline
\end{tabular}

*Amostras: A1) ovo:cenoura 1:1, A2) ovo:cenoura 1:1,5, A3) ovo:cenoura 1:2, A4) ovo: cenoura 1:2,5, A5) ovo:cenoura 1:3, A6) ovo:couve $1: 1$, A7) ovo:couve 1,15 , A8) ovo: couve $1: 2$, A9) ovo:couve $1: 2,5$, A10) ovo:couve $1: 3$, A11) ovo, A12) cenoura, A13) couve; ${ }^{* *}$ Carboidratos totais obtidos por diferença; ${ }^{1}$ Média \pm desvio padrão; ${ }^{2}$ desvio padrão de todas as médias da coluna é igual a zero; $\mathrm{e}^{3}$ Letras diferentes na vertical indicam diferenç̧a significativa entre os tratamentos em nível de $5 \%$.

TABELA 2 - Médias das misturas de cenoura e de couve para análise de umidade ( $\mathrm{g} / 100 \mathrm{~g})$, extrato etéreo $(\mathrm{g} / 100 \mathrm{~g})$, proteínas (g/100 g) e cinzas ( $\mathrm{g} / 100 \mathrm{~g})$.

\begin{tabular}{|c|c|c|c|c|}
\hline \multirow[t]{2}{*}{$\begin{array}{l}\text { Média das } \\
\text { misturas }\end{array}$} & Umidade & $\begin{array}{l}\text { Extrato } \\
\text { etéreo }\end{array}$ & Proteínas & Cinzas \\
\hline & \multicolumn{4}{|c|}{$(\mathrm{g} / 100 \mathrm{~g})$} \\
\hline Cenoura & $91,69^{a 1}$ & $1,47^{b}$ & $2,04^{b}$ & $0,80^{\mathrm{b}}$ \\
\hline Couve & $91,12^{b}$ & $1,59^{a}$ & $2,65^{a}$ & $0,95^{a}$ \\
\hline
\end{tabular}

${ }^{1}$ Letras diferentes na vertical indicam diferença significativa entre os tratamentos em nível de $5 \%$.

apresentaram valores mais elevados de umidade do que as amostras com couve (Tabela 2), sendo que a amostra 5 (3c) apresentou os maiores teores de umidade, e a amostra 6 (1cv) os menores teores (Tabela 1).

Os teores de extrato etéreo das amostras de cenoura e couve se apresentaram dentro da normalidade (Tabela 1) conforme os valores registrados na literatura para cenoura $0,19 \mathrm{~g}, 0,20 \mathrm{~g} \mathrm{e} 0,23 \mathrm{~g} / 100 \mathrm{~g}$ e para a couve $0,28 \mathrm{~g}, 0,46 \mathrm{~g} \mathrm{e}$ $0,34 \mathrm{~g} / 100 \mathrm{~g}[13,14,28]$.

Os valores de extrato etéreo na amostra de ovo (A11) se apresentaram inferiores (Tabela 1) quando comparados com a literatura, devido ao teor de umidade encontrado neles. Dados da literatura registram 10,80 g, 11,29 g, 11,50 g e $10,54 \mathrm{~g} / 100 \mathrm{~g}$ de ovos $[4,12,36,44]$. Os teores mais elevados de extrato etéreo foram encontrados nas amostras com couve e nas maiores proporções de ovo nas misturas (Tabelas 2 e 3 , respectivamente).

Os teores observados (Tabela 1) para proteínas nos ovos foram inferiores aos valores observados na literatura, sendo registrados $12,50 \mathrm{~g}, 13,85 \mathrm{~g}, 12,90 \mathrm{~g}$ e $12,90 \mathrm{~g} / 100 \mathrm{~g}$ de ovo $[4,14,26,44]$, devido à umidade mais elevada ( $\mathrm{Ta}$ bela 1). Para as amostras de cenoura (A12) e couve (A13), os conteúdos de proteínas se apresentaram dentro da nor-
TABELA 3 - Médias das diferentes proporções utilizadas nas misturas (ovo/cenoura e ovo/couve) para os teores de umidade (g/100 g), extrato etéreo (g/100 g), proteínas (g/100 g) e cinzas (g/100 g).

\begin{tabular}{lllll}
\hline Proporções & Umidade & $\begin{array}{c}\text { Extrato } \\
\text { etéreo }\end{array}$ & Proteínas & Cinzas \\
\cline { 2 - 5 } & \multicolumn{4}{c}{$(\mathbf{g} / \mathbf{1 0 0} \mathbf{~ g )}$} \\
\hline $1: 1$ & $89,75^{\mathrm{e} 1}$ & $2,24^{\mathrm{a} 2}$ & $2,92^{\mathrm{a}}$ & $0,91^{\mathrm{a}}$ \\
$1: 1,5$ & $90,84^{\mathrm{d}}$ & $1,73^{\mathrm{b}}$ & $2,70^{\mathrm{b}}$ & $0,89^{\mathrm{ab}}$ \\
$1: 2$ & $91,67^{\mathrm{c}}$ & $1,41^{\mathrm{c}}$ & $2,30^{\mathrm{c}}$ & $0,88^{\mathrm{b}}$ \\
$1: 2,5$ & $92,17^{\mathrm{b}}$ & $1,19^{\mathrm{d}}$ & $1,95^{\mathrm{d}}$ & $0,86^{\mathrm{b}}$ \\
$1: 3$ & $92,59^{\mathrm{a}}$ & $1,04^{\mathrm{e}}$ & $1,83^{\mathrm{e}}$ & $0,82^{\mathrm{c}}$ \\
\hline
\end{tabular}

${ }^{1}$ Letras diferentes na vertical indicam diferença significativa entre os tratamentos em nível de $5 \%$.

malidade, segundo alguns autores que encontraram 0,49 g e 0,68 g/100 g para a cenoura, e 1,40 g e 1,03 g/100 g para couve [13, 28], embora SINGH et al. [42] tenham encontrado valores mais elevados de proteína para a cenoura e couve, sendo $1,19 \mathrm{~g} \mathrm{e} 3,60 \mathrm{~g} / 100 \mathrm{~g}$, respectivamente.

Quando comparadas misturas de cenoura e de couve, foi observado (Tabela 2) que as misturas com couve apresentaram valores mais elevados de proteínas, devido à couve apresentar $1,10 \%$ de proteína, enquanto a cenoura apresenta metade deste teor $(0,55 \%)$. Da mesma forma que os teores de extrato etéreo, as amostras com maior proporção de ovo, apresentaram maiores conteúdos de proteínas, por ser o ovo um alimento que contém substancial concentração deste nutriente, que é encontrado tanto na clara $(10,44 \mathrm{~g} / 100 \mathrm{~g})$ quanto na gema $(15,71 \mathrm{~g} / 100 \mathrm{~g})$ [44].

Os teores de cinzas nas amostras A11, A12 e A13, observados na Tabela 1 , se apresentaram dentro da normalidade, sendo encontrados valores de 0,72 g, 0,58 g e 0,63 g/100 g para ovos; 0,59 g, 0,48 g e 0,56 g/100 g para cenouras; e $0,72 \mathrm{~g}, 0,54 \mathrm{~g}$ e $0,81 \mathrm{~g} / 100 \mathrm{~g}$ para couve, segundo FRANCO [13], IBGE [14] e SALINAS [36], respectivamente. Considerando-se as misturas, os maiores teores de cinza foram encontrados nas amostras com couve (Tabela 2) e, entre as proporções, foram observados valores mais elevados nas amostras com maiores teores de ovo (1:1) e valores mais baixos nas amostras com maiores teores de cenoura e couve (1:3), como pode ser observado na Tabela 3 .

Os valores para carboidratos totais encontrados nas amostras analisadas (Tabela 1) se apresentaram dentro da normalidade, quando comparados com os registrados na literatura, sendo encontrados para os ovos $2,19 \mathrm{~g} \mathrm{e}$ $2,08 \mathrm{~g} / 100 \mathrm{~g}[14,36]$; para a cenoura 3,02 g e 2,79 g/100 g; e para a couve $2,63 \mathrm{~g} \mathrm{e} 3,06 \mathrm{~g} / 100 \mathrm{~g}[13,14]$. Os alimentos utilizados apresentam baixos teores desse nutriente.

\section{2 - Fatores antinutricionais}

Os valores encontrados para os fatores antinutricionais, nas misturas analisadas, são descritos na Tabela 4 .

Os teores de taninos para a couve foram superiores aos registrados na literatura, pois MOSHA et al. [29] encontraram 0,42 mEqcatequina/g. Os teores podem variar de acordo com o tipo de cultivo, a germinação da planta, o grau 
TABELA 4 - Quantidade de taninos em mEqcatequina/g, fitato, em $\mathrm{mg} / \mathrm{g}$ e ácido oxálico em porcentagem, nas misturas analisadas.

\begin{tabular}{lccc}
\hline Amostras $^{*}$ & $\begin{array}{c}\text { Tanino } \\
\text { (mEqcatequina/g) }\end{array}$ & $\begin{array}{c}\text { Fitato } \\
(\mathbf{m g} / \mathbf{g})\end{array}$ & $\begin{array}{c}\text { Ácido Oxálico } \\
(\%)\end{array}$ \\
\hline A1 (1 c) & $1,10^{1 \mathrm{e} 2}$ & $0,41^{\mathrm{a}}$ & $0,06^{\mathrm{f}}$ \\
A2 $(1,5 \mathrm{c})$ & $1,40^{\mathrm{a}}$ & $0,42^{\mathrm{a}}$ & $0,10^{\text {def }}$ \\
A3 $(2 \mathrm{c})$ & $1,32^{\mathrm{b}}$ & $0,41^{\mathrm{a}}$ & $0,10^{\text {def }}$ \\
A4 $(2,5 \mathrm{c})$ & $1,34^{\mathrm{b}}$ & $0,40^{\mathrm{a}}$ & $0,12^{\text {cd }}$ \\
A5 $(3 \mathrm{c})$ & $1,33^{\mathrm{b}}$ & $0,40^{\mathrm{a}}$ & $0,15^{\mathrm{bc}}$ \\
A6 $(1 \mathrm{cv})$ & $1,05^{\mathrm{f}}$ & $0,23^{\mathrm{de}}$ & $0,28^{\mathrm{a}}$ \\
A7 $(1,5 \mathrm{cv})$ & $1,17^{\mathrm{d}}$ & $0,23^{\mathrm{de}}$ & $0,30^{\mathrm{a}}$ \\
A8 $(2 \mathrm{cv})$ & $1,18^{\mathrm{d}}$ & $0,25^{\mathrm{bcd}}$ & $0,10^{\text {def }}$ \\
A9 $(2,5 \mathrm{cv})$ & $1,21^{\text {cd }}$ & $0,27^{\mathrm{bc}}$ & $0,11^{\text {cde }}$ \\
A10 (3 cv) & $1,23^{\mathrm{c}}$ & $0,29^{\mathrm{b}}$ & $0,12^{\text {cd }}$ \\
A11 (o) & $0,0^{\mathrm{i}}$ & $0,0^{\mathrm{g}}$ & $0,0^{\mathrm{g}}$ \\
A12 (c) & $0,78^{\mathrm{h}}$ & $0,17^{\mathrm{f}}$ & $0,09^{\text {def }}$ \\
A13 (cv) & $0,85^{\mathrm{g}}$ & $0,20^{\text {ef }}$ & $0,19^{\mathrm{b}}$ \\
\hline
\end{tabular}

*Amostras: A1) ovo:cenoura 1:1, A2) ovo:cenoura 1:1,5, A3) ovo:cenoura 1:2, A4) ovo: cenoura 1:2,5, A5) ovo:cenoura 1:3, A6) ovo:couve 1:1, A7) ovo:couve 1,15, A8) ovo: couve $1: 2$, A9) ovo:couve $1: 2,5$, A10) ovo:couve $1: 3$, A11) ovo, A12) cenoura, A13) couve; ${ }^{1}$ Desvio padrão de todas as médias da coluna é igual a zero; $\mathrm{e}^{2}$ Letras diferentes na vertical indicam diferença significativa entre os tratamentos em nível de $5 \%$.

de maturação, bem como as condições de processamento e estocagem dos alimentos vegetais [11]. Os taninos têm sido estudados pela formação de complexos insolúveis com o ferro não heme no lúmen intestinal [37], podendo também reagir com outras substâncias.

Na Tabela 5, observou-se que os maiores valores para taninos foram encontrados nas misturas com cenoura. As amostras A7, A8, A9 e A10, com maior conteúdo de couve, também apresentaram teores elevados (Tabela 4).

Em relação às proporções analisadas, observamos que nas médias referentes às proporções $1: 1,5 ; 1: 2,5$ e 1:3, ou seja, nas misturas com maiores quantidades de cenoura e couve, foram encontrados valores mais elevados de taninos (Tabela 6).

TABELA 5 - Médias das misturas de cenoura e de couve para teores de taninos (mEqcatequina/g), fitatos ( $\mathrm{mg} / \mathrm{g}$ ) e oxalato (\%).

\begin{tabular}{cccc}
\hline $\begin{array}{c}\text { Média das } \\
\text { misturas }\end{array}$ & $\begin{array}{c}\text { Taninos } \\
\text { (mEqcatequina/g) }\end{array}$ & $\begin{array}{c}\text { Fitatos } \\
(\mathbf{m g} / \mathbf{g})\end{array}$ & $\begin{array}{c}\text { Oxalato } \\
\mathbf{( \% )}\end{array}$ \\
\hline Cenoura & $1,29^{\mathrm{a} 1}$ & $0,40^{\mathrm{a}}$ & $0,11^{\mathrm{b}}$ \\
Couve & $1,17^{\mathrm{b}}$ & $0,26^{\mathrm{b}}$ & $0,18^{\mathrm{a}}$ \\
\hline
\end{tabular}

${ }^{1}$ Letras diferentes na vertical indicam diferença significativa entre os tratamentos em nível de $5 \%$.

TABELA 6 - Médias das diferentes proporções utilizadas nas misturas (ovo/cenoura e ovo/couve) para os teores de taninos (mEqcatequina/g), fitatos (mg/g) e oxalato (\%) nas diferentes proporções (ovo/cenoura e ovo/couve).

\begin{tabular}{lclc}
\hline Proporções & $\begin{array}{c}\text { Taninos } \\
\text { (mEqcatequina/g) }\end{array}$ & $\begin{array}{c}\text { Fitatos } \\
(\mathbf{m g} / \mathbf{g})\end{array}$ & $\begin{array}{c}\text { Oxalato } \\
(\%)\end{array}$ \\
\hline $1: 1$ & $1,07^{\mathrm{c}}$ & $0,32^{\mathrm{c}}$ & $0,17^{\mathrm{b}}$ \\
$1: 1,5$ & $1,29^{\mathrm{a}}$ & $0,32^{\mathrm{bc}}$ & $0,20^{\mathrm{a}}$ \\
$1: 2$ & $1,25^{\mathrm{b}}$ & $0,33^{\mathrm{abc}}$ & $0,10^{\mathrm{d}}$ \\
$1: 2,5$ & $1,28^{\mathrm{a}}$ & $0,34^{\mathrm{ab}}$ & $0,12^{\mathrm{cd}}$ \\
$1: 3$ & $1,28^{\mathrm{a}}$ & $0,35^{\mathrm{a}}$ & $0,14^{\mathrm{c}}$ \\
\hline
\end{tabular}

${ }^{1}$ Letras diferentes na vertical indicam diferença significativa entre os tratamentos em nível de $5 \%$.
Em relação aos fitatos (Tabela 4), os teores mais altos foram identificados nas amostras contendo cenoura (A1 a A5). Os valores de fitatos para cenoura (A12) foram semelhantes aos apresentados por SINGH et al. [42], 0,18 mg/g.

Na Tabela 6 , pode ser observada diferença significativa entre os valores de fitatos para as diferentes proporções analisadas, visto que quanto maior a quantidade de couve ou cenoura maior a quantidade de fitatos.

Para o ácido oxálico, a couve (A13) foi o alimento com a maior quantidade deste fator antinutricional (Tabela 4), embora os valores encontrados tenham sido inferiores a $0,72 \%$, registrados na pesquisa realizada por BOAVENTURA [6]. Segundo este autor, os teores de ácido oxálico nos alimentos podem variar com a época do ano e tratos culturais. Por outro lado, MOSHA et al. [29] não encontraram teores de ácido oxálico na couve, após 10 min de cocção doméstica.

Em relação às misturas analisadas, foram identificados maiores teores de ácido oxálico nas amostras com couve (Tabela 5), sendo que nas proporções 1:1,5 foram encontrados valores mais elevados, seguido das proporções $1: 1$, como pode ser observado na Tabela 6 .

\section{3 - $\alpha$ - e $\beta$-caroteno}

A Tabela 7 mostra o conteúdo em $\mu \mathrm{g} / 100 \mathrm{~g}$ de alfa e beta caroteno das amostras e o valor de vitamina A total em $\mu \mathrm{g} / 100 \mathrm{~g}$ de Retinol Equivalente (RE) e em $\mu \mathrm{g} / 100 \mathrm{~g}$ de Atividade de Retinol Equivalente (RAE).

Os valores encontrados para o $\alpha$-caroteno na amostra de cenoura (A12) se apresentaram dentro da normalidade quando comparados com THANE \& REDDY [43], que identificaram teores de $4.400 \mu \mathrm{g} / 100 \mathrm{~g}$ de $\alpha$-caroteno em cenouras fatiadas cozidas. Entre as misturas com cenoura, a amostra 4 (2,5 c) apresentou o melhor resultado para o $\alpha$-caroteno, e a amostra 1 ( $1 \mathrm{c}$ ) apresentou os valores mais baixos.

Os valores de $\beta$-caroteno para a cenoura foram superiores quando comparados com alguns trabalhos, pois GODOY \& RODRIGUES-AMAYA [18] e SINGH et al. [42] encontraram $3,32 \mathrm{mg}$ e 2,2 mg/100 g, respectivamente. Foram semelhantes ao valor encontrado por HÄAG et al. [20], que observou diferenças consideráveis nos teores de $\beta$-caroteno em cenouras cultivadas em diversas regiões com diferentes níveis de latitude; e foram inferiores aos valores registrados por THANE \& REDDY [43]; que encontraram $8.800 \mu \mathrm{g} / 100 \mathrm{~g}$ de $\beta$-caroteno em cenouras fatiadas cozidas. Em relação à couve, os teores encontrados também foram superiores, pois alguns autores registraram valores entre 0,08 e 4,86 mg/100 g para o $\beta$-caroteno [22, 23].

Entre as misturas analisadas (Tabela 8), as amostras com cenoura apresentaram valores mais elevados de $\beta$-caroteno do que as amostras com couve, sendo que as amostras A4 (2,5 c) e A10 (3 cv) apresentaram os maiores valores, e as amostras A6 ( $1 \mathrm{cv}$ ) e A7 ( $1,5 \mathrm{c}$ ), os valores mais baixos (Tabela 7). 
TABELA 7 - Média dos valores de alfa caroteno, beta caroteno e equivalente de retinol das amostras.

\begin{tabular}{|c|c|c|c|c|}
\hline \multirow[t]{2}{*}{ Amostras* } & $\alpha$-Caroteno & $\beta$-Caroteno & $\begin{array}{l}\mathrm{RE}^{* *} \\
\text { Total }\end{array}$ & $\begin{array}{c}\mathrm{RAE}^{* * *} \\
\text { Total }\end{array}$ \\
\hline & \multicolumn{4}{|c|}{$(\mu \mathrm{g} / 100 \mathrm{~g})$} \\
\hline $\mathrm{A} 1$ (1 c) & $2435,27 \pm 8,73^{1 \mathrm{e} 2}$ & $3496,06 \pm 3,93^{e}$ & 348,61 & 697,22 \\
\hline $\mathrm{A} 2(1,5 \mathrm{c})$ & $4038,90 \pm 65,41^{c}$ & $5421,52 \pm 67,78^{\mathrm{cd}}$ & 562,47 & 1124,94 \\
\hline A3 (2 c) & $3884,66 \pm 11,68^{d}$ & $5429,68 \pm 25,16^{c d}$ & 549,95 & 1099,91 \\
\hline A4 $(2,5 \mathrm{c})$ & $4368,53 \pm 35,08^{b}$ & $5360,49 \pm 4,63^{c d}$ & 587,40 & 1174,80 \\
\hline A5 (3 c) & $3902,64 \pm 36,09^{d}$ & $5602,98 \pm 9,30^{\circ}$ & 558,68 & 1117,36 \\
\hline A6 $(1 \mathrm{cv})$ & n.d. & $2406,75 \pm 19,74^{f}$ & 100,28 & 200,56 \\
\hline A7 (1,5 cv) & n.d. & $2782,25 \pm 67,02^{f}$ & 115,93 & 231,85 \\
\hline A8 (2 cv) & n.d. & $3508,52 \pm 61,64^{e}$ & 146,19 & 292,38 \\
\hline A9 $(2,5 \mathrm{cv})$ & n.d. & $3605,69 \pm 80,99^{\mathrm{e}}$ & 150,24 & 300,47 \\
\hline A10 (3 cv) & n.d. & $7894,08 \pm 8,46^{a}$ & 328,92 & 657,84 \\
\hline $\mathrm{A} 11$ (o) & n.d. & $105,37 \pm 7,14^{g}$ & 4,39 & 8,78 \\
\hline $\mathrm{A} 12$ (c) & $4609,83 \pm 63,19^{a}$ & $6605,58 \pm 68,07^{b}$ & 659,39 & 1318,77 \\
\hline $\mathrm{A} 13(\mathrm{cv})$ & n.d. & $5169,16 \pm 52,85^{c}$ & 215,38 & 430,76 \\
\hline
\end{tabular}

TABELA 8 - Médias das misturas de cenoura e de couve para análise de $\beta$-Caroteno ( $\mu \mathrm{g} / 100 \mathrm{~g})$, ferro total $(\mathrm{mg} / 100 \mathrm{~g})$ e ferro dialisável (\%).

\begin{tabular}{cccc}
\hline $\begin{array}{c}\text { Média das } \\
\text { misturas }\end{array}$ & $\begin{array}{c}\beta-C a r o t e n o \\
(\mu \mathbf{g} / \mathbf{1 0 0} \mathbf{~ g})\end{array}$ & $\begin{array}{c}\text { Ferro total } \\
(\mathbf{m g} / \mathbf{1 0 0} \mathbf{~ g})\end{array}$ & $\begin{array}{c}\text { Ferro dialisável } \\
(\%)\end{array}$ \\
\hline Cenoura & $5296,86^{\mathrm{a}}$ & $0,55^{\mathrm{b} 1}$ & $2,45^{\mathrm{a} 1}$ \\
Couve & $4036,95^{\mathrm{b}}$ & $1,01^{\mathrm{a}}$ & $1,97^{\mathrm{b}}$ \\
\hline
\end{tabular}

${ }^{1}$ Letras diferentes na vertical indicam diferença significativa entre os tratamentos em nível de $5 \%$.

Sendo consideradas as diferentes proporções (ovo/cenoura e ovo/couve), foi constatado que aumentando as quantidades de cenoura e couve nas misturas, há aumento significativo nos teores de $\beta$-caroteno nas amostras analisadas, por serem estes alimentos boas fontes deste carotenóide (Tabela 9).

Os valores de Retinol Equivalente (RE) para a cenoura (Tabela 7) foram inferiores aos registrados na literatura, pois SAUNDERS et al. [40] encontraram valores de $1.677,5 \mu \mathrm{g} / 100 \mathrm{~g}$, e ENDEF [14] e FRANCO [13] encontraram, respectivamente, $1.100 \mu \mathrm{g}$ e $900 \mu \mathrm{g} \mathrm{RE} / 100 \mathrm{~g}$.

TABELA 9 - Médias das diferentes proporções utilizadas nas misturas (ovo/cenoura e ovo/couve) para os teores de $\beta$-Caroteno $(\mu \mathrm{g} / 100 \mathrm{~g})$, ferro total $(\mathrm{mg} / 100 \mathrm{~g})$ e ferro dialisável (\%).

\begin{tabular}{llcc}
\hline Proporções & $\begin{array}{c}\beta \text {-Caroteno } \\
(\boldsymbol{\mu} \mathbf{g} / \mathbf{1 0 0} \mathbf{~ g})\end{array}$ & $\begin{array}{c}\text { Ferro total } \\
(\mathbf{m g} / \mathbf{1 0 0} \mathbf{~ g})\end{array}$ & $\begin{array}{c}\text { Ferro dialisável } \\
(\mathbf{\%})\end{array}$ \\
\hline $1: 1$ & $2951,41^{\mathrm{e}}$ & $0,94^{\mathrm{a}}$ & $1,88^{\mathrm{c}}$ \\
$1: 1,5$ & $4101,88^{\mathrm{d}}$ & $0,82^{\mathrm{b}}$ & $1,51^{\mathrm{d}}$ \\
$1: 2$ & $4450,85^{\mathrm{c}}$ & $0,64^{\mathrm{d}}$ & $1,96^{\mathrm{c}}$ \\
$1: 2,5$ & $5088,11^{\mathrm{b}}$ & $0,77^{\mathrm{c}}$ & $2,30^{\mathrm{b}}$ \\
$1: 3$ & $6742,27^{\mathrm{a}}$ & $0,73^{\mathrm{cd}}$ & $3,39^{\mathrm{a}}$ \\
\hline
\end{tabular}

${ }^{1}$ Letras diferentes na vertical indicam diferença significativa entre os tratamentos em nível de $5 \%$.
Em relação à couve, os valores observados para o Retinol Equivalente (RE) também foram inferiores aos encontrados na literatura, FRANCO [13] registrou $750 \mu \mathrm{g} / 100 \mathrm{~g}$ e ENDEF [14] $650 \mu \mathrm{g} / 100 \mathrm{~g}$. Entre as misturas analisadas, numericamente as amostras com couve apresentaram os teores mais baixos de Retinol Equivalente (RE).

\section{4 - Ferro nos alimentos}

Os resultados para o ferro total nas amostras analisadas podem ser observados na Tabela 10.

TABELA 10 - Ferro total (mg/100 g) e porcentagem de ferro dialisável nas amostras analisadas.

\begin{tabular}{|c|c|c|}
\hline Amostras* & Ferro total $(\mathrm{mg} / 100 \mathrm{~g})$ & Ferro dialisável (\%) \\
\hline $\mathrm{A} 1(1 \mathrm{c})$ & $0,68 \pm 0,01^{1 \mathrm{~cd}}$ & $2,18 \pm 0,1^{\text {cde }}$ \\
\hline $\mathrm{A} 2(1,5 \mathrm{c})$ & $0,61 \pm 0,01^{\text {cde }}$ & $1,71 \pm 0,1^{\text {def }}$ \\
\hline A3 (2c) & $0,54 \pm 0,01^{\text {def }}$ & $1,93 \pm 0,0^{\text {cde }}$ \\
\hline A4 $(2,5 \mathrm{c})$ & $0,49 \pm 0,01^{\text {ef }}$ & $2,41 \pm 0,07^{\text {cde }}$ \\
\hline A5 (3 c) & $0,45 \pm 0,02^{f}$ & $4,04 \pm 0,2^{b}$ \\
\hline A6 $(1 \mathrm{cv})$ & $1,21 \pm 0,10^{a}$ & $1,58 \pm 0,1^{\text {ef }}$ \\
\hline A7 (1,5 cv) & $1,04 \pm 0,02^{b}$ & $1,30 \pm 0,03^{\text {ef }}$ \\
\hline A8 $(2 \mathrm{cv})$ & $0,75 \pm 0,05^{c}$ & $1,99 \pm 0,11^{\text {cdef }}$ \\
\hline A9 $(2,5 \mathrm{cv})$ & $1,05 \pm 0,04^{b}$ & $2,20 \pm 0,11^{\text {cdef }}$ \\
\hline $\mathrm{A} 10(3 \mathrm{cv})$ & $1,01 \pm 0,04^{b}$ & $2,75 \pm 0,05^{\mathrm{cd}}$ \\
\hline $\mathrm{A} 11$ (o) & $1,33 \pm 0,14^{a}$ & $0,99 \pm 0,09^{f}$ \\
\hline $\mathrm{A} 12$ (c) & $0,25 \pm 0,00^{g}$ & $7,43 \pm 0,12^{\mathrm{a}}$ \\
\hline $\mathrm{A} 13(\mathrm{cv})$ & $0,63 \pm 0,02^{\text {cde }}$ & $3,31 \pm 0,12^{c}$ \\
\hline
\end{tabular}

*Amostras: A1) ovo:cenoura 1:1, A2) ovo:cenoura 1:1,5, A3) ovo:cenoura 1:2, A4) ovo: cenoura $1: 2,5$, A5) ovo:cenoura $1: 3$, A6) ovo:couve $1: 1$, A7) ovo:couve $1,15, \mathrm{~A} 8$ ) ovo: couve $1: 2$, A9) ovo:couve $1: 2,5$, A10) ovo:couve $1: 3$, A11) ovo, A12) cenoura, A13) couve ${ }^{1}$ Média \pm desvio padrão; e ${ }^{2}$ Letras diferentes na vertical indicam diferença significativa entre os tratamentos em nível de $5 \%$.

Pode ser observado que o ovo é um alimento considerado fonte de ferro, portanto as amostras com maiores proporções de ovo apresentaram, numericamente, maiores teores de ferro, sendo a cenoura um alimento pobre neste mineral. Porém, os valores encontrados para as amostras de ovo e cenoura foram inferiores aos registrados na literatura, sendo encontrados para os ovos 2,41 mg, 3,2 mg e 2,3 mg/100 g [ $14,36,42]$ e para a cenoura $0,6 \mathrm{mg}$ e $0,7 \mathrm{mg} / 100 \mathrm{~g}[26,36]$, e somente a amostra de couve apresentou valores dentro da normalidade, sendo encontrados $0,5 \mathrm{mg}, 2,2 \mathrm{mg}$ e $0,65 \mathrm{mg} / 100 \mathrm{~g}[13,14,28]$.

Na Tabela 8, observamos que entre as misturas analisadas, o teor de ferro total mostrou-se mais expressivo nas amostras com couve e, entre as diferentes proporções, os teores mais elevados foram encontrados nas proporções 1:1 e 1:1,5, ou seja, nas amostras com maiores quantidades de ovo nas misturas (Tabela 9).

É importante conhecer o teor de ferro total dos alimentos, mesmo que não seja uma quantia que caracterize sua efetiva utilização pelo organismo, devido ao conteúdo de substâncias estimuladoras e inibidoras presentes. A partir destes teores, identificam-se as fontes naturais deste elemento entre os alimentos disponíveis. 


\section{5 - Quantidade de ferro dialisável}

Foi observado que houve diferença no ferro dialisável nas diferentes amostras analisadas (Tabela 10). As misturas de cenoura apresentaram os teores mais elevados de ferro dialisável quando comparadas com as amostras com couve (Tabela 8) e, entre as proporções, foi observado que aumentando as quantidades de cenoura e couve (proporções 1:2,5 e 1:3) nas amostras, ocorreu aumento significativo nos teores do ferro dialisável (Tabela 9).

A amostra A5 (3 c) apresentou o melhor valor de ferro dialisável, seguida pelas amostras A1, A3, A4, A8, A9 e A10.

Na Tabela 11, podem ser observados os valores estatísticos de correlação entre os teores de ferro dialisável e os outros componentes nas misturas analisadas.

TABELA 11 - Valores estatísticos de correlação para os teores de ferro dialisável e os demais nutrientes entre as diferentes amostras analisadas.

\begin{tabular}{lll}
\hline \multicolumn{1}{c}{ Variáveis } & \multicolumn{1}{c}{ Valor de $\mathbf{R}^{2}$} & \multicolumn{1}{c}{ Valor de F } \\
\hline Ferro dialisável x Umidade & 0,511964249 & $3,06604 \mathrm{E}-07^{\mathrm{s}}$ \\
Ferro dialisável x Extrato Etéreo & 0,332307422 & $0,000122649^{\mathrm{s}}$ \\
Ferro dialisável x Proteínas & 0,39860204 & $1,63548 \mathrm{E}-05^{\mathrm{s}}$ \\
Ferro dialisável x Cinzas & 0,36595275 & $4,51701 \mathrm{E}-05^{\mathrm{s}}$ \\
Ferro dialisável x Fitatos & 0,069722706 & $0,10430764^{\mathrm{ns}}$ \\
Ferro dialisável x Oxalatos & 0,04916296 & $0,174911351^{\mathrm{ns}}$ \\
Ferro dialisável x Taninos & 0,004559681 & $0,682948682^{\mathrm{ns}}$ \\
Ferro dialisável x Beta caroteno & 0,318882257 & $0,000180475^{\mathrm{s}}$ \\
\hline
\end{tabular}

ns = correlação não significativa entre os tratamentos em nível de $5 \%$ de probabilidade; e $\mathrm{s}=$ correlação significativa entre os tratamentos em nível de $5 \%$ de probabilidade.

Pode ser observado que, em relação à umidade, extrato etéreo, proteínas, cinzas e $\beta$-caroteno, houve correlação significativa para o ferro dialisável, sendo que para umidade e $\beta$-caroteno essa correlação foi positiva e para os demais componentes foi negativa.

Segundo BENITO \& MILLER [5], há interferência da gordura na absorção do ferro, como foi observado nos resultados (Tabela 11), sendo que o teor de gordura também pode influenciar na disponibilidade do $\beta$-caroteno, como constatado por RIEDL et al. [34].

GERMANO [17] identificou em seu estudo que as misturas com ovo aumentaram a disponibilidade do ferro, principalmente pelo conteúdo expressivo de proteínas e extrato etéreo. Segundo BENITO \& MILLER [5], o principal mecanismo da absorção do ferro no duodeno é por um processo, dependente de energia, por meio de um carreador envolvendo proteína transportadora através da membrana. De acordo com GARCIA et al. [16], os aminoácidos liberados no processo de digestão das proteínas formam quelatos com o ferro não heme, facilitando sua absorção no lúmen intestinal.

Porém, analisando as misturas de alimentos em diferentes proporções, ocorreu correlação negativa significativa entre os valores de extrato etéreo e proteínas com o ferro dialisável, pois à medida que os teores destes componentes foram diminuindo nas misturas, foram observados valores mais elevados para o ferro dialisável. GARCIA et al. [16], citam em seus estudos que o efeito das proteínas sobre a absorção do ferro não heme é diferenciado de acordo com a fonte alimentar, tendo as proteínas de carnes, aves e peixes efeito estimulador, e as proteínas de leite e ovos, efeito inibidor, ou ainda, estas não tem demonstrado efeito interferente sobre a absorção do ferro de alimentos vegetais.

Por outro lado, HEATH \& FAIRWEATHER-TAIT [21] relatam em seus trabalhos que alguns peptídios contendo aminoácido cisteína apresentam efeito facilitador da absorção do ferro, e os ovos contêm em média $290 \mathrm{mg}$ de cisteína/100 g. Porém, GARCIA et al. [16] relatam que o efeito estimulador da cisteína é observado quando este aminoácido é administrado na forma de peptídios ou em cápsulas de gelatina, mas não quando é consumido em misturas de alimentos, devido à alcalinidade provocada por muitos alimentos vegetais que promovem a oxidação da cisteína para cistina.

Em relação aos fatores antinutricionais não houve correlação significativa entre seus valores com o ferro dialisável (Tabela 11).

As amostras com cenouras apresentaram os valores mais elevados de fitato (Tabela 5) e teores elevados de ferro dialisável (Tabela 8). Segundo DOMINGUEZ et al. [10], os efeitos dos fitatos sobre a disponibilidade dos minerais dependem de um número expressivo de fatores, entre os quais se destacam a concentração de fitatos nos alimentos e sua capacidade de complexação com os diferentes minerais; o teor de proteínas na dieta, e, portanto, da presença de peptídios e aminoácidos no intestino que podem interferir na formação do complexo fitato-mineral; além da presença de outros agentes quelantes, como fibra dietética, ácido oxálico, ácido cítrico e taninos, que podem competir com os fitatos em sua complexação com os minerais.

As amostras com cenoura apresentaram teores superiores de fitato, mas também teores elevados de $\beta$-caroteno (Tabela 8 ), o que pode sugerir que o $\beta$-caroteno tenha protegido o ferro dos efeitos interferentes dos fitatos, pois segundo LAYRISSE et al. [24, 25], tem sido pesquisado que tanto a vitamina A quanto o seu precursor, o $\beta$-caroteno, são capazes de interferir no efeito inibitório que têm os fitatos e polifenóis na absorção do ferro não heme, sendo que o $\beta$-caroteno é ainda mais eficiente que a vitamina $A$ neste ponto.

O $\beta$-caroteno apresentou correlação positiva significativa com o ferro dialisável (Tabela 11). Na amostras A4 ( 2,5 c) e A5 (3 c), que apresentaram valores altos para o ferro dialisável, foram encontrados também teores elevados de beta caroteno, assim como para a amostra A10 (3 cv), que também apresentou valores numericamente maiores para o ferro dialisável. Ao contrário, na amostra A6 (1 cv), cujos valores de ferro total foram altos e os valores de ferro dialisável foram baixos, foram também obtidos teores baixos de beta caroteno. 
O ovo é um alimento considerado fonte de ferro, porém sua disponibilidade, quando analisada a amostra pura (A11), foi baixa. HEATH \& FAIRWEATHER-TAIT [21], apontam em seus estudos que a avidina, glicoproteína presente nos ovos, é um componente responsável pela inibição da absorção do ferro não-heme pelo organismo.

Já na cenoura (A12), embora com pequena quantia de ferro, a biodisponibilidade deste mineral foi elevada, assim como os valores de $\beta$-caroteno. Desta forma, pode ser observado que, utilizando diferentes proporções de ovo e cenoura, é possível obter quantia maior de ferro biodisponível, pelo efeito estimulador do $\beta$-caroteno.

\section{4 - CONCLUSÓES}

As amostras com cenoura apresentaram os teores mais elevados de ferro dialisável, visto que sendo aumentadas as quantidades de cenoura e couve nas amostras, foi constatado aumento significativo nos teores do ferro dialisável.

O extrato etéreo e as proteínas apresentaram correlação negativa significativa com o ferro dialisável, sendo observado aumento do ferro dialisável com a redução dos teores destes componentes nas misturas analisadas.

Não houve interferência significativa dos fatores antinutricionais sobre o ferro dialisável, embora os taninos e ácido oxálico tenham atuado como fatores limitantes quando observados os valores numéricos nas amostras com maiores concentrações de cenoura e couve.

O $\beta$-caroteno apresentou correlação positiva significativa com o ferro dialisável, exercendo influência na redução do efeito dos antinutricionais, especialmente dos fitatos, na disponibilidade do ferro, principalmente nas amostras com cenoura.

Pode ser recomendada a utilização de misturas de alimentos como ovo e cenoura, sendo que este último deve estar em maior proporção em refeições destinadas a populações carentes em ferro.

\section{5 - REFERÊNCIAS BIBLIOGRÁFICAS}

[1] ASSOCIATION OF OFFICIAL ANALYTICAL CHEMISTS. Official methods of analysis of the Association of Official Analytical Chemists. $16^{\circ}$ ed. Washington: AOAC, 1995. 2 v.

[2] BATISTA FILHO, M.; FERREIRA, L. O. C. Prevenção e tratamento da anemia nutricional ferropriva: novos enfoques e perspectivas. Caderno de Saúde Pública, v. 12, n. 3, p. S181- S191, 1996.

[3] BEARD, J. L.; DAWSON, H.; PIÑERO, D. J. Iron metabolism: a comprehensive review. Nutrition Reviews, v. 54, n. 10, p. 295-317, 1996.

[4] BEDOGNI, G.; BATTISTINI, N. C. Effects of cooking and storage on the nutritional value of eggs. In: Watson, R. R. ed. Eggs and health promotion, cap. 17 p. 177-183. Iowa State Press, 2002.

[5] BENITO, P.; MILlER, D. M. Iron Absorption and Bioavailability: an updated review. Nutrition Research, v. 18, n. 3, p. 581-603, 1998.
[6] BOAVEnTURA, A. C. Avaliação química, protéica e biodisponibilidade de cálcio nas folhas de couve-manteiga, couve-flor e espinafre. Piracicaba, 1998. 46p. Dissertação (M. S.) - Escola Superior de Agricultura "Luiz de Queiroz", Universidade de São Paulo.

[7] CABALLERO, B. Interaciones entre los componentes de la dieta. Archivos Latinoamericanos de Nutrición, v. 38, n. 3, p. 656-684, 1988.

[8] COMPANHIA DE ENTREPOSTOS E ARMAZÉNS GERAIS DE SÃO PAULO - CEAGESP. www.ceagesp.com. br/temporad.htm (09 ago. 2004).

[9] DINIZ, A. S. Combate à deficiência de vitamina A: linhas de ação e perspectivas. Revista Brasileira de Saúde materno Infantil, v. 1, n. 1, p. 31-36, 2001.

[10] DOMínGUEZ, B. M.; GÓMEZ, M. V. I.; LEÓN, F. R. Ácido fítico: aspectos nutricionales e implicaciones analíticas. Archivos Latinoamericanos de Nutrición. v. 52, n. 3, p. 219-231, 2002.

[11] DREWNOWSKI, A.; GOMEZ-CARNEROS, C. Bitter taste, phytonutrients, and the consumer: a review. American Journal of Clinical Nutrition, v. 72, n. 6, p. 1424-35, 2000.

[12] ENGLERT, S. Avicultura: tudo sobre raças, manejo e nutrição. $7^{\circ}$ ed. Guaíba: Livraria e Editora Agropecuária, 1998, 238p.

[13] FRANCO, G. Tabela de composição química dos alimentos. $8^{\circ}$ ed. Rio de Janeiro: Atheneu, 1992. 230p.

[14] FUNDAÇÃO INSTITUTO BRASILEIRO DE GEOGRAFIA E ESTATÍSTICA. Estudo Nacional de Despesa Familiar. Tabela de Composição de Alimentos, $2^{\circ}$ ed. Rio de Janeiro: FIBGE, 1996, 213p.

[15] FUNDAÇÃO INSTITUTO BRASILEIRO DE GEOGRAFIA E ESTATÍSTICA. Banco de Dados Agregados. www.sidra.ibge.gov.br/bda/horti/default.asp (10 ago. 2004).

[16] GARCIA, M. N.; MARTINEZ-TORRES, C.; LEETS, I. et al. Heat tratament on heme iron and iron-containing proteins in meat: Iron absorption in humans from diets containing cooked meat fractions. Nutritional Biochemistry, v. 7, n.1, p. 49-54, 1996.

[17] GERMANO, R. M. A. Disponibilidade de ferro na presença do $\beta$-Caroteno e o efeito dos interferentes em combinações de alimentos. Piracicaba, 2002. 95p. Dissertação (M. S.) - Escola Superior de Agricultura "Luiz de Queiroz", Universidade de São Paulo.

[18] GODOY, H. T.; RODRIGUES-AMAYA, D. B. Ocurrence of cis isomers of provitamins A in Brazilian vegetables. Journal Agricultural of Food Chemistry, v. 46, n. 8, p. 3081-3086, Aug. 1998.

[19] GRYNSPAN, F.; CHERYAN, M. Phytate - calcium interations with soy protein. Journal of the American Oil Chemistry Society, v. 66, n. 1, p. 93-97, 1989.

[20] HÄAG, M.; YLIKOSKI, S.; KUMPULAINEN, J. Vitamin C and $\alpha$ - and $\beta$-carotene contents in vegetables consumed in Finland during 1988-1989 and 1992-1993. Journal of Food Composition and Analysis, v. 7, p. 252-259, 1994.

[21] HEATH, A. L. M.; FAIRWEATHER-TAIT, S. J. Clinical implications of changes in the modern diet: iron intake, absorption and status. Best Practice \& Research Clinical Haematology, v. 15, n. 2, p. 225-241, 2002. 
[22] KIMURA, M.; RODRIGUES-AMAYA, D. B.; GODOY, H. T. Assessment of saponification of step in the quantitative determination of carotenoids and provitamins A. Food Chemistry, v. 35, n. 3, p. 187-195, 1990.

[23] KURILICH, A. C.; TSAU, G. J.; BROWN, A. et al. Carotene, tocopherol and ascorbate contentes in subspecies of Brassica oleracea. Journal Agricultural of Food Chemistry, v. 47, n. 4, p. 1576-1581, Apr. 1999.

[24] LAYRISSE, M.; GARCÍA-CASAL, M. N,; SOLANO, L. et al. The role of vitamin A on the inhibitor of nonheme iron absorption: Preliminary results. Jornal of Nutritional Biochemistry, v. 8, n.2, p. 61-67, 1997.

[25] LAYRISSE, M.; GARCIA-CASAL, M. N; SOLANO, L. et al. Iron bioavailability in humans from breakfasts enriched with iron bis-glycine chelate, phytates and polyphenols. Journal of Nutrition, v. 130, n. 9, p. 2195-2199, Sep. 2000.

[26] MENDEZ, M. H. M.; DERIVI, S. C. N.; RODRIGUES, M. C. R. et al. Tabela de Composição de Alimentos. Niterói: Editora da Universidade Federal Fluminense, 1995. 39p.

[27] MOIR, K. W. Determination of oxalic acid in plant Queensland. Journal of Agriculture Science, v. 10, n. 1, p. 1-3, Mar. 1953.

[28] MOSHA, T. C; GAGA, H. E. Nutritive value and effect of blanching on the trypsin and chymotrypsin inhibitor activities of selected leafy vegetables. Plant foods for Human Nutrition, v. 53, n. 3, p. 271-283, 1999.

[29] MOSHA, T. C.; GAGA, H. E.; PACE, R. D. et al. Effect of blanching on the content of antinutritional factors in selected vegetables. Plant Foods for Human Nutrition, v. 47, n. 4, p. 361-367, Oct. 1995.

[30] NAS/NRC (NATIONAL ACADEMY OF SCIENCES / NATIONAL COUNCIL RESEARCH). Recommended Dietary Allowances. $9^{\circ}$ ed. Washington, D. C., 1980. p. 51-71.

[31]NATIONAL RESEARCH COUNCIL/ Dietary Reference Intakes: for Vitamin A, Vitamin K, Arsenic, Boron, Cromium, Copper, Iodine, Iron, Manganese, Molybdenium, Nickel, Silicon, Vanadium and Zinc. Washington, D.C., National Academy Press, 2001, 797p.

[32] OPAS/OMS. Micronutrient deficiencies. Battling iron deficiency anaemia. http://www.who.int/nut/ida.htm (15 out. 2002)

[33] PRICE, M. L.; HAGERMAN, A. E.; BUTLER, L. G. Tannin content of cowpeas, chickpeas, pigeon peas and mung beans. Journal of Agricultural and Food Chemistry, v. 28 , n. 2 , p. $459-461,1980$.

[34] RIEDL, J.; LINSEISEN, J.; HOFFMAN, J; WOLFRAM, G. Some Dietary Fibers Reduce the Absorption of Caro- tenoids in Women. Journal of Nutrition, v. 129, n.12, p. 2170-2179, 1999.

[35] RODRIGUES, R. S. M.; PENTEADO, M. V. C. Carotenóides com atividade Pró-vitamínica A em hortaliças folhosas. Revista de Farmácia e Bioquímica da Universidade de São Paulo, v. 25, n. 1, p. 39-52, 1989.

[36] SALINAS, R. D. Alimentos e Nutrição: Introdução a Bromatologia. $3^{\circ}$ ed. Porto Alegre: Artmed, 2002. 278p.

[37] SAMMAN, S.; SANDSTRÖM, B; TOFT, M. B. et al. Green tea or rosemary extract added to foods reduces nonheme-iron absorption. American Journal of Clinical Nutrition, v. 73, n. 3, p. 607-612, 2001.

[38] SARRUGE, J. R.; HAAG, H. P.; Análises químicas em plantas. Piracicaba, ESALQ/USP, 1974. 56p.

[39] SAS STATISTICAL ANALYSIS SYSTEM INSTITUTE. Sas/Qc software; usage and reference. $2^{\circ}$ ed. Cary, 1996. $2 \mathrm{v}$.

[40] SAUNDERS, C.; RAMALHO, A.; ACCILY, E. et al. Utilização de tabelas de composição de alimentos na avaliação do risco de hipovitaminose A. Archivos Latinoamericanos de Nutrición, v. 50, n. 3, p. 237-242, 2000.

[41] SILVA, J. D. Análise de Alimentos (métodos químicos e biológicos). Viçosa: UFV, Imprensa Universitária, 1981. 166p.

[42] SINGH, G.; KAWATRA, A.; SEHGAL, S. Nutritional composition of selected green leafy vegetables, herbs and carrots. Plant Foods for Human Nutrition, v. 56, n. 4, p. 359-364, 2001.

[43] THANE, C.; REDDY, S. Processing of fruit and vegetables: effect on carotenoids. Nutrition \& Food Science, v. 97 n. 2, p. 58-65, 1997.

[44] TORRES, A. F. S.; CAMPOS, N. C.; DUARTE, M. L.G.; PHILIPPI, S. T.; RODRIGUES, R. S. M. Composição Centesimal e Valor calórico de alimentos de origem animal. Ciência e Tecnologia de Alimentos, v. 20, n. 2, p. 145-150, 2000 .

[45] WHITTAKER, P.; FOX, M. R. S; FORBES, A. L. In vitro prediction of iron bioavailability for food fortification. Nutrition Reports International, v. 39, n. 6 , p. 1205-1215, 1989.

\section{6 - AGRADECIMENTOS}

À Técnica Maria de Lourdes Perin Storer do Laboratório de Nutrição Humana e Alimentos pelo auxílio na realização das análises químicas. 